Proceedings of the

International Geometry Center

Vol. 10, no. 1 (2017) pp. 22-46

\title{
Homeotopy groups of one-dimensional foliations on surfaces
}

\author{
Sergiy Maksymenko, Eugene Polulyakh, Yuliya Soroka
}

\begin{abstract}
Let $Z$ be a non-compact two-dimensional manifold obtained from a family of open strips $\mathbb{R} \times(0,1)$ with boundary intervals by gluing those strips along their boundary intervals. Every such strip has a foliation into parallel lines $\mathbb{R} \times t, t \in(0,1)$, and boundary intervals, whence we get a foliation $\Delta$ on all of $Z$. Many types of foliations on surfaces with leaves homeomorphic to the real line have such "striped" structure. That fact was discovered by W. Kaplan (1940-41) for foliations on the plane $\mathbb{R}^{2}$ by level-set of pseudo-harmonic functions $\mathbb{R}^{2} \rightarrow \mathbb{R}$ without singularities.

Previously, the first two authors studied the homotopy type of the group $\mathcal{H}(\Delta)$ of homeomorphisms of $Z$ sending leaves of $\Delta$ onto leaves, and shown that except for two cases the identity path component $\mathcal{H}_{0}(\Delta)$ of $\mathcal{H}(\Delta)$ is contractible. The aim of the present paper is to show that the quotient $\mathcal{H}(\Delta) / \mathcal{H}_{0}(\Delta)$ can be identified with the group of automorphisms of a certain graph with additional structure encoding the "combinatorics" of gluing.
\end{abstract}

\section{INTRODUCTION}

Let $Z$ be a non-compact two-dimensional manifold and $\Delta$ be a onedimensional foliation on $Z$ such that each leaf $\omega$ of $\Delta$ is homeomorphic to $\mathbb{R}$ and is a closed subset of $Z$. These foliations on the plane often appear as level-sets of pseudoharmonic functions and from that point of view they were studied by W. Kaplan [6], [7], W. Boothby [1], [2], M. Morse and J. Jenkins [5], M. Morse [11] and others.

In particular, Kaplan proved that for every such a foliation there exists at most countably many leaves $\left\{\omega_{i}\right\}_{i \in J}$ such that for every connected component $S$ of $\mathbb{R}^{2} \backslash\left\{\omega_{i}\right\}_{i \in J}$ one can find a homeomorphism $\phi: S \rightarrow \mathbb{R}^{2} \times(0,1)$ sending the leaves in $S$ onto horizontal lines $\mathbb{R} \times\{t\}, i \in(0,1)$. However his construction was not canonical, as he tried to minimize the total number of strips, and for that reason the closure $\bar{S}$ may have very complicated

2010 Mathematics Subject Classification: 57R30, 55P15

Keywords: foliations, striped surface 
structure. For instance the above homeomorphism $\phi$ not always extends to an embedding of $\bar{S}$ into $\mathbb{R} \times[0,1]$.

In [9, Theorem 1.8] the first two authors gave sufficient conditions for a one-dimensional foliation on a non-compact surface to have a similar striped structure, and proposed a certain canonical decomposition into strips whose closures homeomorphic to open subsets of $\mathbb{R} \times[0,1]$.

Also, in [8] the same authors considered arbitrary foliated surfaces $(Z, \Delta)$ glued from strips in the above way and studied the homotopy properties of the group of homeomorphisms $\mathcal{H}(\Delta)$ of $Z$ mapping leaves of the foliation $\Delta$ into leaves. They proved that except for few cases the identity path component $\mathcal{H}_{0}(\Delta)$ of $\mathcal{H}(\Delta)$ is contractible. The principal technical assumption in [8] was that the gluing maps between boundary intervals of strips must be affine.

The quotient $\pi_{0} \mathcal{H}(\Delta)=\mathcal{H}(\Delta) / \mathcal{H}_{0}(\Delta)$ is an analogue of a mapping class group for foliated homeomorphisms and we call it the homeotopy group of the foliation $\Delta$. In [12] and [13] the third author studied a special class of so-called "rooted tree like" striped surfaces, completely described algebraic structure of homeotopy groups of their foliations, and also related those groups with the homeotopy groups of the space of leaves $Z / \Delta$.

The aim of the present paper is to extend the results of [8] to arbitrary "striped" surfaces and compute the corresponding homeotopy groups. Namely, we show that $\pi_{0} \mathcal{H}(\Delta)$ is isomorphic to a group of automorphism of a certain graph with additional structure, see Theorem 8.1. In particular, these results hold for all foliations considered in [9], [12], [13].

Structure of the paper. $\S 2$ contains a list of the principal results of the paper. First we give a formal definition of a strip and then show in Proposition 2.2 that up to a foliated homeomorphism it can be replaced by a model strip having better disposition of boundary intervals.

Next, we characterize homeomorphisms between boundaries of strips which extend to foliated homeomorphisms between strips, see Theorem 2.3. $\S 3$ and $\S 4$ are devoted to proofs of those results.

In $\S 5$ we introduce a notion of a striped atlas on a surface $Z$, being a decomposition into strips glued along boundary intervals, and prove that gluing homeomorphisms can be made affine, see Theorem 5.8.

Further, in $\S 6$, we associate to each striped atlas a certain graph $G$ which encodes the "combinatorics" of gluing strips, and relate automorphisms of $G$ with self-equivalences of the corresponding atlas, see Theorem 6.2.

$\S 7$ establishes relationships between distinct properties of foliated surfaces considered in [8], [9], and [10], see Theorem 7.4.

Finally, in $\S 8$ we consider the group $\mathcal{H}(\Delta)$ of homeomorphisms of the foliation $\Delta$ and deduce from [8] and results of previous sections that the 
homeotopy group $\pi_{0} \mathcal{H}(\Delta)$ is isomorphic with the group of automorphisms of the graph associated to some special striped atlas of $Z$.

\section{MODEL STRIPS}

Let $Z$ be a two-dimensional topological manifold. A foliated chart of dimension 1 on $Z$ is a pair $(U, \varphi)$, where $U \subset Z$ is an open subset and $\varphi: U \rightarrow(a, b) \times B$ is a homeomorphism with $B$ being an open subset of $[0,+\infty)$. The set $P_{y}=\varphi^{-1}((a, b) \times\{y\}), y \in B$, is then called a plaque of this foliated chart.

Suppose $\Delta=\left\{\omega_{\alpha} \mid \alpha \in A\right\}$ is a partition of $Z$ into path connected subsets and there exists an atlas $\mathcal{A}=\left\{U_{i}, \varphi_{i}\right\}_{i \in \Lambda}$ of foliated charts of dimension 1 on $Z$ such that for each $\alpha \in A$ and each $i \in \Lambda$ every path component of a set $\omega_{\alpha} \cap U_{i}$ is a plaque. Then $\Delta$ is said to be a one-dimensional foliation on $Z$ and $\left\{U_{i}, \varphi_{i}\right\}_{i \in \Lambda}$ is called a foliated atlas associated to $\Delta$. Every $\omega_{\alpha}$ is then a leaf of the foliation $\Delta$ and the pair $(Z, \Delta)$ is a foliated surface.

Let $\left(Z_{1}, \Delta_{1}\right)$ and $\left(Z_{2}, \Delta_{2}\right)$ be two foliated surfaces. Then a homeomorphism $h: Z_{1} \rightarrow Z_{2}$ is said to be foliated if for each leaf $\omega \in \Delta_{1}$ its image, $h(\omega)$, is a leaf of $\Delta_{2}$.

Definition 2.1. A subset $S \subset \mathbb{R}^{2}$ will be called a strip if

(i) $\mathbb{R} \times(u, v) \subset S \subset \mathbb{R} \times[u, v]$;

(ii) $S$ is open in the topology of $\mathbb{R} \times[u, v]$

for some $u<v \in \mathbb{R}$. Denote

$$
\begin{aligned}
\partial_{-} S & :=S \cap \mathbb{R} \times\{u\}, & & \partial_{+} S:=S \cap \mathbb{R} \times\{v\}, \\
\partial S & :=\partial_{-} S \cup \partial_{+} S, & & \operatorname{Int} S:=\mathbb{R} \times(u, v) .
\end{aligned}
$$

We will call $\partial S$ the boundary of $S$, while $\partial_{-} S$ and $\partial_{+} S$ will be the sides of $S$. It follows that $\partial S$ is an open subset of $\mathbb{R} \times\{u, v\}$, and so it is a disjoint union of at most countably many open (possibly unbounded) intervals.

If, in addition to (i) and (ii), the following conditions hold:

(iii) every connected component of $\partial S$ is a bounded interval,

(iv) the closures of boundary intervals of $\partial S$ in $\mathbb{R} \times[u, v]$ are mutually disjoint,

then $S$ will be called a model strip.

Evidently, each strip $S$ possesses an oriented one-dimensional foliation into horizontal lines $\mathbb{R} \times t, t \in(u, v)$ and boundary intervals of $\partial S$. We will call that foliation canonical.

The following statement allows to reduce any strip to a technically more convenient form. It will be proved in Section 3.

Proposition 2.2. Each strip is foliated homeomorphic to a model strip. 
Monotone homeomorphisms of $\partial S$. Notice that the boundary of a strip can be regarded as a partially ordered set being a disjoint union of two linearly ordered sets $\partial_{-} S$ and $\partial_{+} S$ that are incomparable with each other. In other words, for $(a, x),(b, y) \in \partial S$ we assume that $(a, x)<(b, y)$ if and only if $a<b$ and $x=y$.

More generally, let $A, B \subset \partial S$ be two subsets. Then we say that $A<B$ if and only if $a<b$ for all $a \in A$ and $b \in B$. In particular, this gives a linear order on the boundary intervals of $\partial_{-} S$ and $\partial_{+} S$. Thus, if $I_{\alpha}=(a, b) \times\{x\}$ and $I_{\beta}=(c, d) \times\{y\}$ are boundary intervals of $\partial S$ with $x, y \in\{u, v\}$, then $I_{\alpha}<I_{\beta}$ if and only if $x=y$ and $b<c$.

Now let $S_{1}$ and $S_{2}$ be two strips, $A \subset \partial S_{1}$ and $B \subset \partial S_{2}$ be subsets, and $h: A \rightarrow B$ be a bijection. We will say that $h$ preserves (resp. reverses) order whenever for any $a, a^{\prime} \in A$ we have that $a<a^{\prime}$ if and only if $h(a)<h\left(a^{\prime}\right)$ (resp. $h(a)>h\left(a^{\prime}\right)$ ). In either of these cases $h$ is said to be monotone.

Evidently, if $h: S_{1} \rightarrow S_{2}$ is a foliated homeomorhism between two strips, then its restriction $\left.h\right|_{\partial S_{1}}: \partial S_{1} \rightarrow \partial S_{2}$ is monotone. The following statement is a converse to the latter observation. It will be proved in Section 4.

Theorem 2.3. Every monotone homeomorphism $h: \partial S_{1} \rightarrow \partial S_{2}$ between boundaries of two strips $S_{1}$ and $S_{2}$ extends to a foliated homeomorphism $\hat{h}: S_{1} \rightarrow S_{2}$.

\section{Proof of Proposition 2.2}

Lemma 3.1. Let $S$ be a half strip with $\mathbb{R} \times[0,1) \subset S \subset \mathbb{R} \times[0,1]$. Then there exists a half strip $S^{\prime}$ and foliated homeomorphism $h: S \rightarrow S^{\prime}$ such that

- $\mathbb{R} \times[0,1) \subset S^{\prime} \subset S \subset \mathbb{R} \times[0,1]$;

- the closures of boundary intervals of $\partial_{+} S^{\prime}$ are bounded in $\mathbb{R}^{2}$ and mutually disjoint;

- $h$ is fixed on $\mathbb{R} \times 0$;

- $h$ preserves the second coordinate, and therefore is foliated.

Assuming lemma is true let us deduce Proposition 2.2. Let $S$ be a strip with $\operatorname{Int}(S)=\mathbb{R} \times(-1,1)$. Consider two half strips

$$
A=S \cap(\mathbb{R} \times[-1,0]), \quad B=S \cap(\mathbb{R} \times[0,1]) .
$$

Then by Lemma 3.1 one can find two half strips $A^{\prime}$ and $B^{\prime}$ and foliated homeomorphisms $f: A \rightarrow A^{\prime}$ and $g: B \rightarrow B^{\prime}$ such that

- $\mathbb{R} \times(-1,0] \subset A^{\prime} \subset A \subset \mathbb{R} \times[-1,0]$;

- $\mathbb{R} \times[0,1) \subset B^{\prime} \subset B \subset \mathbb{R} \times[0,1]$;

- the closures of boundary intervals of $\partial_{-} A^{\prime}$ and $\partial_{+} B^{\prime}$ are bounded in $\mathbb{R}^{2}$ and mutually disjoint; 
- $f$ and $g$ are fixed on $\mathbb{R} \times 0$ and preserve second coordinate.

Then $S^{\prime}=A^{\prime} \cup B^{\prime}$ is a model strip with $\mathbb{R} \times[0,1) \subset S^{\prime} \subset S$, and a foliated homeomorphism $h: S \rightarrow S^{\prime}$ can be given by the formula: $\left.h\right|_{A^{\prime}}=f$ and $\left.h\right|_{B^{\prime}}=g$. This proves Proposition 2.2 modulo Lemma 3.1.

Proof of Lemma 3.1. a) First we will show how to make closures of boundary intervals of $\partial_{+} S^{\prime}$ to be bounded though not necessarily disjoint. Fix any $a<b \in \mathbb{R}$ and consider the following half strip:

$$
T=\mathbb{R} \times[0,1) \cup(a, b) \times\{1\} .
$$

Then by [8, Lemma 3.2] there exists a homeomorphism $h: \mathbb{R} \times[0,1] \rightarrow T$ preserving second coordinate and fixed on $\mathbb{R} \times 0$. Hence $S^{\prime}=h(S)$ is a half strip with $\partial S^{\prime} \subset \partial T=(a, b) \times 1$.

b) To simplify the notation replace $S$ with $S^{\prime}$ and assume that boundary intervals of $\partial_{+} S^{\prime}$ are bounded in $\mathbb{R}^{2}$. We should make their closures mutually disjoint.

Consider the following subset of $\mathbb{R}^{7}$ :

$$
A=\{(a, u, b, c, v, d, t) \mid a<u<b, c<v<d, t \in[a, b]\}
$$

and define the function $\gamma: A \rightarrow \mathbb{R}$ by

$$
\gamma(a, u, b, c, v, d, t)= \begin{cases}\frac{t-a}{u-a}(v-c)+c, & t \in[a ; u], \\ \frac{t-u}{b-u}(d-v)+v, & t \in[u ; b] .\end{cases}
$$

Then $\gamma$ is continuous, and for any combination of the first six parameters $a, u, b, c, v, d$ the map $t \mapsto \gamma(a, u, b, c, v, d, t)$ homeomorphically maps the segment $[a, b]$ onto $[c, d]$ so that $u$ is sent to $v$.

Lemma 3.1.1. Let $T$ be a closed triangle in the plane $\mathbb{R}^{2}$ with vertices $A\left(x_{a}, y_{a}\right), B\left(x_{b}, y_{b}\right), O\left(x_{o}, y_{o}\right)$ such that $y_{a}=y_{b}>y_{o}$, and $C$ be a point on the open interval $(A, B)$. Let also

$$
T^{\prime}=T \backslash\{A, B\}, \quad T^{\prime \prime}=T \backslash\{A \cup[B, C]\} .
$$

Then there exists a homeomorphism $f: T^{\prime} \rightarrow T^{\prime \prime}$ preserving second coordinate. In particular, $f$ is fixed on the sides $(A, O],[O, B)$ and maps the interval $(A, B)$ onto $(A, C)$.

Proof. Not loosing generality one can assume that $A(-1,0), B(1,1), O(0,0)$ and $C(0.5,1)$. Then $f$ can be given by the formula:

$$
f(x, y)= \begin{cases}\left(\gamma\left(-y, y^{2}, y,-y, 0, y, x\right), y\right), & 0 \leqslant y<1 \\ \left(\frac{x+1}{2}-1,1\right), & y=1 .\end{cases}
$$


Evidently, $f$ maps the curve $x=y^{2}$ on the segment of the line $x=0$. Moreover, we have that $f\left[A_{y}, Q_{y}\right]=\left[A_{y}, C_{y}\right]$, and $f\left[Q_{y}, B_{y}\right]=\left[C_{y}, B_{y}\right]$, see Figure 3.1,
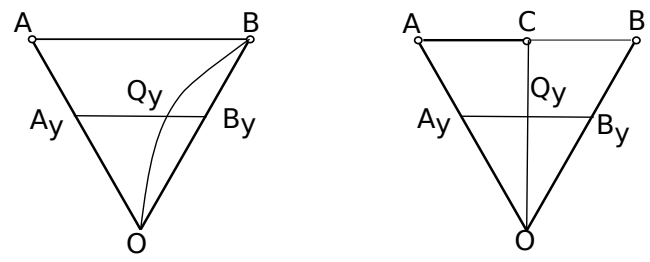

FiguRE 3.1. Triangles $T^{\prime}$ and $T^{\prime \prime}$

Returning back to the proof of Lemma 3.1 assume that

$$
\partial_{+} S=\bigsqcup_{i=1}^{N}\left(a_{i}, b_{i}\right) \times\{1\},
$$

where $N$ is either a finite number of $+\infty$. Fix a strictly monotone sequence $\left\{u_{j}\right\}_{j=1}^{\infty} \subset[-1,1]$ such that $\lim _{j \rightarrow \infty}=1$ and for each $i$ define the triangle $T_{i}$ with vertices $A_{i}\left(a_{i}, 1\right), B_{i}\left(b_{i}, 1\right), O_{i}\left(\frac{a_{i}+b_{i}}{2}, u_{i}\right)$, and let $C\left(\frac{a_{i}+b_{i}}{2}, 1\right)$. Define also the following model half strips:

$$
S_{i}=S \backslash \bigcup_{j \leq i}\left[C_{j}, B_{j}\right) \times\{1\}
$$

and put

$$
S_{0}=S, \quad S^{\prime}=S \backslash \bigcup_{i=1}^{N}\left[C_{i}, B_{i}\right) \times\{1\}=\bigcap_{i=1}^{N} S_{i} .
$$

Then the closures of the boundary intervals of $S^{\prime}$ are mutually disjoint.

Denote $T_{i}^{\prime}=T_{i} \backslash\left\{A_{i}, B_{i}\right\}$ and $T_{i}^{\prime \prime}=T_{i} \backslash\left\{A_{i} \cup\left[B_{i}, C_{i}\right]\right\}$. Then by Lemma 3.1.1 there exists a homeomorphism $f_{i}: T_{i}^{\prime} \rightarrow T_{i}^{\prime \prime}$ preserving second coordinate and being identity on the sides $\left(A_{i}, O_{i}\right]$ and $\left[O_{i}, B_{i}\right)$. Therefore $f_{i}$ extends by the identity to a homeomorphism $f_{i}: S_{i-1} \rightarrow S_{i}$.

Then a foliated homeomorphism $\varphi: S \rightarrow S^{\prime}$ can be defined as the composition of all $f_{i}$ :

$$
\varphi=\cdots \circ f_{i+1} \circ f_{i} \circ \cdots \circ f_{2} \circ f_{1}: S=S_{0} \stackrel{f_{1}}{\longrightarrow} S_{1} \stackrel{f_{2}}{\longrightarrow} \cdots \stackrel{f_{i}}{\longrightarrow} S_{i} \stackrel{f_{i+1}}{\longrightarrow} \cdots S^{\prime} .
$$

If $N$ is finite, $\varphi$ is well-defined.

For infinite $N$ one should check that for each point $z \in S$ the sequence

$$
\left\{g_{j}=f_{j} \circ \cdots \circ f_{2} \circ f_{1}: S \rightarrow S_{j} \subset S\right\}_{j \in \mathbb{N}}
$$


of embeddings "stabilizes" on some neighborhood $U_{z}$ of $z$, that is $g_{j}=g_{j+1}$ on $U$ for all sufficiently large $j$.

So, let $z=(x, y) \in S$. If $y<1$, then there exists $i$ such that $y<u_{i}$. Let $U=\mathbb{R} \times\left(0, u_{i}\right)$. Then $f_{j}(U)=U$ for all $j$. Moreover, $f_{j}$ is fixed on $U$ for all $j>i$. Therefore $g_{j}=g_{i}$ on $U$ for all $j>i$.

Suppose $y=1$, so $(x, y) \in\left(a_{i}, b_{i}\right) \times\{1\}$ for some $i$, then each $f_{j}$ with $j \neq i$ is fixed on the triangle $T_{i}^{\prime}$. Then $U=T_{i}^{\prime} \backslash\left(\left(A_{i}, O_{i}\right] \cup\left[O_{i}, B_{i}\right)\right)$ is an open neighborhood of $(x, y)$ in $S$, and $g_{j}=g_{i}=f_{i}$ on $U$ for $j>i$. Thus $\varphi$ is a homeomorphism. Lemma 3.1 is completed.

\section{Proof of Theorem 2.3}

Let $S_{1}$ and $S_{2}$ be two strips and $h: \partial S_{1} \rightarrow \partial S_{2}$ a monotone homeomorphism. We should prove that $h$ extends to a foliated homeomorphism between $S_{1}$ and $S_{2}$.

If $\partial S_{1}$, and so $\partial S_{2}$, are empty, then any foliated homeomorphism between $S_{1}$ and $S_{2}$ can be regarded as an extension of $h$. Therefore we will suppose that $\partial S_{1} \neq \varnothing$. Not loosing generality one can also assume that $h\left(\partial_{-} S_{1}\right)=\partial_{-} S_{2}, h\left(\partial_{+} S_{1}\right)=\partial_{+} S_{2}$, and the restrictions $\left.h\right|_{\partial_{-} S_{1}}$ and $\left.h\right|_{\partial_{+} S_{1}}$ are increasing.

Case 1. Suppose that both $S_{1}$ and $S_{2}$ are half strips such that

$$
\begin{gathered}
\partial_{+} S_{1}=\bigsqcup_{i=1}^{K} X_{i} \times\{1\}, \quad \partial_{+} S_{2}=\bigsqcup_{i=1}^{K} X_{i}^{\prime} \times\{1\}, \\
\partial_{-} S_{1}=\partial_{-} S_{2}=\mathbb{R} \times\{0\},
\end{gathered}
$$

where $K \in\{0,1, \ldots,+\infty\}$, each $X_{i} \times\{1\}$ is a boundary interval of $\partial_{+} S_{1}$, $X_{i}^{\prime}$ is a boundary interval of $\partial_{+} S_{2}$, the closures $\overline{X_{i}}$ and $\overline{X_{i}^{\prime}}$ are bounded,

$$
\overline{X_{i}} \cap \overline{X_{j}}=\varnothing, \quad \overline{X_{i}^{\prime}} \cap \overline{X_{j}^{\prime}}=\varnothing,
$$

for all $i \neq j$, and $h\left(X_{i} \times\{1\}\right)=X_{i}^{\prime} \times\{1\}$.

We will extend $h$ to a homeomorphism $h: S_{1} \rightarrow S_{2}$ preserving second coordinate and fixed on $\mathbb{R} \times 0$.

Fix an arbitrary strictly increasing sequence $\left\{u_{j}\right\}_{j=0}^{\infty} \subset[0,1)$ such that $u_{0}=0$ and $\lim _{j \rightarrow \infty} u_{j}=1$. For each $u_{j}=0,1, \ldots, \infty$ we will now construct a homeomorphism $\psi_{j}: \mathbb{R} \rightarrow \mathbb{R}$ by the following rule.

Since $h(\mathbb{R} \times 0)=\mathbb{R} \times 0$ one can write $h(x, 0)=\left(\psi_{0}(x), 0\right)$ for a unique homeomorphism $\psi_{0}: \mathbb{R} \rightarrow \mathbb{R}$.

Further notice that there exists a unique homeomorphism

$$
\bar{h}: \bigsqcup_{i=1}^{K} X_{i} \rightarrow \bigsqcup_{i=1}^{K} X_{i}^{\prime}
$$


such that $\bar{h}\left(X_{i}\right)=X_{i}^{\prime}$. Then for $j \geq 1$ define $\psi_{j}: \bigsqcup_{i=1}^{j} X_{i} \rightarrow \bigsqcup_{i=1}^{j} X_{i}^{\prime}$ by $\psi_{j}(x)=\bar{h}(x)$. The assumption that $h$ preserves the order of boundary intervals means that $X_{i}<X_{j}$ if and only if $X_{i}^{\prime}<X_{j}^{\prime}, i, j \in 1, \ldots, K$. Hence one can apply the following Lemma 4.1 to extend $\psi_{j}$ to a homeomorphism $\psi_{j}: \mathbb{R} \rightarrow \mathbb{R}$.

Lemma 4.1. Let $\alpha=\left\{X_{i}\right\}_{i=1}^{n}$ and $\beta=\left\{X_{i}^{\prime}\right\}_{i=1}^{n}$ be two families of open segments in $\mathbb{R}$ having the following properties:

(1) the closures $\overline{X_{i}}$ and $\overline{X_{i}^{\prime}}$ are bounded, and $\overline{X_{i}} \cap \overline{X_{j}}=\overline{X_{i}^{\prime}} \cap \overline{X_{j}^{\prime}}=\varnothing$ for all $i \neq j=1, \ldots, n$;

(2) $\alpha$ and $\beta$ are "similarly ordered", that is $X_{i}<X_{j}$ if and only if $X_{i}^{\prime}<X_{j}^{\prime}$ for all $i \neq j=1, \ldots, n$.

Suppose also that for each $i=1, \ldots, n$ we have an orientation preserving homeomorphism $\psi_{i}: X_{i} \rightarrow X_{i}^{\prime}$. Then there is a homeomorphism $\psi: \mathbb{R} \rightarrow \mathbb{R}$ such that $\left.\psi\right|_{X_{i}}=\psi_{i}$.

Proof. Due to assumptions on $\alpha$ and $\beta$ one can renumber the elements in these families and assume that $X_{i}=\left(a_{i}, b_{i}\right)$ and $X_{i}^{\prime}=\left(c_{i}, d_{i}\right)$ for some $a_{i}, b_{i}, c_{i}, b_{i} \in \mathbb{R}$ such that

$$
a_{1}<b_{1}<a_{2}<b_{2}<\ldots<a_{n}<b_{n}, \quad c_{1}<d_{1}<c_{2}<d_{2}<\ldots<c_{n}<d_{n} .
$$

Then the homeomorphism $\psi$ can be given by the formula:

$$
\psi(x)= \begin{cases}x-a_{1}+c_{1}, & x \in\left(-\infty, a_{1}\right] \\ \psi_{i}(x), & x \in\left(a_{i}, b_{i}\right), i=1, \ldots, n \\ \frac{c_{i+1}-d_{i}}{a_{i+1}-b_{i}}\left(x-b_{i}\right)+d_{i}, & x \in\left[b_{i}, a_{i+1}\right], i=1, \ldots, n-1, \\ x-b_{n}+d_{n}, & x \in\left[b_{n},+\infty\right)\end{cases}
$$

Lemma 4.1 is proved.

Now define $h: S_{1} \rightarrow S_{2}$ by the formula:

$$
h(x, y)= \begin{cases}\left(\psi_{j}(x), y\right), & y=u_{j}, \\ \left(\varepsilon_{j}(y) \psi_{j}(x)+\left(1-\varepsilon_{j}(y)\right) \psi_{j+1}(x), y\right), & y \leq j \leq K \\ & y \leq\left(u_{j}, u_{j+1}\right) \\ & 0 \leq j \leq K-1\end{cases}
$$

where $\varepsilon_{j}(y)=\frac{u_{j+1}-y}{u_{j+1}-u_{j}}$.

Evidently, $h$ is bijective, preserves the second coordinate and homeomorphically maps $S_{1} \backslash \partial_{+} S_{1}$ onto $S_{2} \backslash \partial_{+} S_{2}$. 
It remains to check that $h$ is a homeomorphism. Since $\psi_{j}=\psi_{j+1}=\bar{h}$ on $X_{i}$ for $i \leq j$, it follows from the second line in (4.2) that

$$
h(x, y)=(\bar{h}(x), y) .
$$

for all $(x, y) \in X_{i} \times\left(u_{i}, 1\right]$. Therefore $h$ homeomorphically maps the open set $X_{i} \times\left(u_{i}, 1\right]$ of $S_{1}$ onto the open set $X_{i}^{\prime} \times\left(u_{i}, 1\right]$ of $S_{2}$. Since the family

$$
\left\{X_{i} \times\left(u_{i}, 1\right]\right\}_{i=1}^{K} \cup\left\{S_{1} \backslash \partial_{+} S_{1}\right\}
$$

constitutes an open covering of $S_{1}$, it follows that $h$ is a homeomorphism.

Case 2. Suppose $S_{1}$ and $S_{2}$ are arbitrary half strips.

One can assume that $\mathbb{R} \times[0,1) \subset S_{i} \subset \mathbb{R} \times[0,1], i=1,2$. Then by Lemma 3.1 one can find a half strip $S_{i}^{\prime}$ and a homeomorphism $\phi_{i}: S_{i} \rightarrow S_{i}^{\prime}$ such that

- $\mathbb{R} \times[0,1) \subset S_{i}^{\prime} \subset S_{i} \subset \mathbb{R} \times[0,1]$,

- the closures of boundary intervals in $\partial_{+} S_{i}^{\prime}$ are bounded and mutually disjoint;

- $\phi_{i}$ is fixed on $\mathbb{R} \times 0$ and preserves the second coordinate.

Hence the composition $h^{\prime}=\phi_{2} \circ h \circ \phi_{1}^{-1}: \partial S_{1}^{\prime} \rightarrow \partial S_{2}^{\prime}$ is a homeomorphism preserving order and orientations of boundary intervals and coincides with $h$ on $\mathbb{R} \times 0$. Therefore, by Case 1, it extends to a foliated homeomorphism $h^{\prime}: S_{1}^{\prime} \rightarrow S_{2}^{\prime}$. Hence $\phi_{2}^{-1} \circ h^{\prime} \circ \phi_{1}: S_{1} \rightarrow S_{2}$ is the required extension of $h$.

Case 3. Consider the general case when $S_{1}$ and $S_{2}$ are strips. Not loosing generality one can assume that

$$
\mathbb{R} \times(-1,1) \subset S_{i} \subset \mathbb{R} \times[-1,1]
$$

for $i=1,2$. Similarly to the proof of Proposition 2.2 consider two half strips

$$
A_{i}=S_{i} \cap \mathbb{R} \times[-1,0], \quad \quad B_{i}=S_{i} \cap \mathbb{R} \times[0,1] .
$$

Evidently,

$$
\partial_{-} A_{i}=\partial_{-} S_{i}, \quad \partial_{+} A_{i}=\partial_{-} B_{i}=\mathbb{R} \times 0, \quad \partial_{+} B_{i}=\partial_{+} S_{i} .
$$

Define two homeomorphisms $f: \partial A_{1} \rightarrow \partial A_{2}$ and $g: \partial B_{1} \rightarrow \partial B_{2}$ by the rule:

$$
\left.f\right|_{\partial_{-} A_{1}}=\left.h\right|_{\partial_{-} A_{1}},\left.\quad f\right|_{\partial_{+} A_{1}}=\left.g\right|_{\partial_{-} B_{1}}=\mathrm{id}_{\mathbb{R} \times 0},\left.\quad f\right|_{\partial_{+} B_{1}}=\left.h\right|_{\partial_{+} S_{1}} .
$$

Then, by Case 2, $f$ and $g$ extend to foliated homeomorphisms $f: A_{1} \rightarrow A_{2}$ and $g: B_{1} \rightarrow B_{2}$. Hence a required extension $h: S_{1} \rightarrow S_{2}$ of $h$ can be given by the formula: $\left.h\right|_{A^{\prime}}=f$ and $\left.h\right|_{B^{\prime}}=g$. Theorem 2.3 is completed. 


\section{StRiped ATLAS}

Let $Z$ be a two-dimensional topological manifold.

Definition 5.1. A striped atlas on $Z$ is a map $q: Z_{0} \rightarrow Z$ having the following properties:

(1) $Z_{0}=\bigsqcup_{\lambda \in \Lambda} S_{\lambda}$ is at most countable family of mutually disjoint strips;

(2) $q$ is a quotient map, which means that it is continuous, surjective, and has the property that a subset $U \subset Z$ is open if and only if $q^{-1}(U) \cap S_{\lambda}$ is open in $S_{\lambda}$ for each $\lambda \in \Lambda$;

(3) there exist two disjoint families $\mathcal{X}=\left\{X_{\gamma}\right\}_{\gamma \in \Gamma}$ and $\mathcal{Y}=\left\{Y_{\gamma}\right\}_{\gamma \in \Gamma}$ of mutually disjoint boundary intervals of $Z_{0}$ enumerated by the same set of indexes $\Gamma$ such that

(a) $q$ is injective on $Z_{0} \backslash(\mathcal{X} \cup \mathcal{Y})$;

(b) $q\left(X_{\gamma}\right)=q\left(Y_{\gamma}\right)$ for each $\gamma \in \Gamma$;

(c) the restrictions $\left.q\right|_{X_{\gamma}}: X_{\gamma} \rightarrow q\left(X_{\gamma}\right)$ and $\left.q\right|_{Y_{\gamma}}: Y_{\gamma} \rightarrow q\left(Y_{\gamma}\right)$ are embeddings with closed images;

Definition 5.2. A surface $Z$ admitting a striped atlas will be called a striped surface.

Notice that a striped surface $Z$ is a non-compact two-dimensional manifold which can be non-connected and non-orientable, and each of its boundary component is an open interval.

Moreover, $Z$ admits a one-dimensional foliation obtained from canonical foliations on the corresponding model strips $S_{\lambda}$. We will call it the canonical foliation associated to the striped atlas $q$ and denote by $\Delta$. Evidently, each leaf of $\Delta$ is a homeomorphic image of $\mathbb{R}$ and is also a closed subset of $Z$.

Definition 5.3. We will say that a foliated surface $(Z, \Delta)$ admits a striped structure if there exists a striped atlas $q: Z_{0} \rightarrow Z$ which maps each leaf of the canonical foliation of each strip in $Z_{0}$ onto some leaf of $\Delta$.

Remark 5.4. Due to (c) for each $\gamma \in \Gamma$ one get the following "gluing" homeomorphism $\phi_{\gamma}: Y_{\gamma} \rightarrow X_{\gamma}$ defined by

$$
\phi_{\gamma}=\left.\left(\left.q\right|_{X_{\gamma}}\right)^{-1} \circ q\right|_{Y_{\gamma}} .
$$

Therefore one can think that a striped surface is obtained from a family of model strips by gluing them along certain boundary intervals by homeomorphisms $\phi_{\gamma}$. It is allowed that two strips are glued along more than one pair of boundary components. Moreover, one may glue together boundary components of the same strip $S$. 
Definition 5.5. Two striped atlases $q: Z_{0} \rightarrow Z$ and $q^{\prime}: Z_{0}^{\prime} \rightarrow Z^{\prime}$ on striped surfaces $Z$ and $Z^{\prime}$ will be called equivalent if there exist two foliated homeomorphisms $h: Z_{0} \rightarrow Z_{0}^{\prime}$ and $k: Z \rightarrow Z^{\prime}$ making commutative the following diagram:

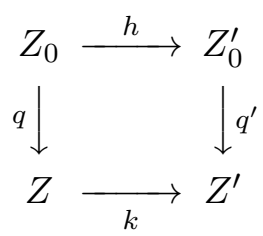

Turning back to the definition of a striped surface notice that for each $\gamma \in \Gamma$ the intervals $X_{\gamma}, Y_{\gamma}$ are horizontal, and so

$$
X_{\gamma}=(a, b) \times\left\{x_{\gamma}\right\}, \quad Y_{\gamma}=(c, d) \times\left\{y_{\gamma}\right\}
$$

for some $x_{\gamma}, y_{\gamma} \in\{u, v\}$ and $a, b, c, d \in \mathbb{R} \cup\{ \pm \infty\}$ with $a<b$ and $c<d$. Hence $\phi_{\gamma}: Y_{\gamma} \rightarrow X_{\gamma}$ can be written as follows:

$$
\phi_{\gamma}\left(s, y_{\gamma}\right)=\left(\psi_{\gamma}(s), x_{\gamma}\right), \quad s \in(c, d),
$$

where $\psi_{\gamma}:(c, d) \rightarrow(a, b)$ is a certain homeomorphism.

Remark 5.6. Notice that if $a<b$ and $c<d$, then there exist exactly two affine homeomorphisms $\psi^{+}, \psi^{-}:(c, d) \rightarrow(a, b)$ given by

$$
\psi^{+}(t)=\frac{b-a}{d-c}(t-c)+a, \quad \psi^{-}(t)=\frac{a-b}{d-c}(t-c)+b,
$$

for $t \in(a, b)$. Evidently, $\psi^{+}$preserves the orientation and $\psi^{-}$reverses it.

Definition 5.7. A striped atlas $q: Z_{0} \rightarrow Z$ will be called affine if the following two conditions hold:

(a) $Z_{0}$ consists of model strips only;

(b) each gluing map $\phi_{\gamma}: Y_{\gamma} \rightarrow X_{\gamma}, \gamma \in \Gamma$, is affine, that is the homeomorphism $\psi_{\gamma}$ in (5.3) is given by either of the formulas from (5.4).

Theorem 5.8. Each striped atlas $q: Z_{0}=\bigsqcup_{\lambda \in \Lambda} S_{\lambda} \rightarrow Z$ on a striped surface $Z$ is equivalent to an affine one. Moreover, if $Z_{0}$ consists of model strips only, then there exists a foliated homeomorphism $h: Z_{0} \rightarrow Z_{0}$ such that the composition $q^{\prime}=q \circ h: Z_{0} \rightarrow Z$ is an affine atlas.

Proof. First we show that $q$ is equivalent to an atlas consisting of model strips only. By Proposition 2.2 for every strip $S_{\lambda}$ there exists a model strip $S_{\lambda}^{\prime} \subset S_{\lambda}$ and a foliated homeomorphism $h_{\lambda}: S_{\lambda}^{\prime} \rightarrow S_{\lambda}$. Put $Z_{0}^{\prime}=\bigsqcup_{\lambda \in \Lambda} S_{\lambda}^{\prime}$ and define a homeomorphism $h: Z_{0}^{\prime} \rightarrow Z_{0}$ by $\left.h\right|_{S_{\lambda}^{\prime}}=h_{\lambda}, \lambda \in \Lambda$. Then 
$q^{\prime}=q \circ h: Z_{0}^{\prime} \rightarrow Z$ is an atlas on $Z$ consisting of model strips and the pair $\left(h, \operatorname{id}_{Z}\right)$ is an equivalence between $q^{\prime}$ and $q$.

Assume now that each strip $S_{\lambda}$ in $Z_{0}$ is model. For each $\gamma \in \Gamma$ let $\sigma_{\gamma}: Y_{\gamma} \rightarrow X_{\gamma}$ be a unique affine homeomorphism preserving or reversing orientation mutually with $\phi_{\gamma}$.

Let $S_{\lambda}, \lambda \in \Lambda$, be a model strip from $Z_{0}$ and $\partial S_{\lambda}=\underset{\alpha \in \mathbf{A}}{\sqcup} I_{\alpha}$ be the family of its boundary intervals. We will now define a certain homeomorphism $h_{\lambda}: \partial S_{\lambda} \rightarrow \partial S_{\lambda}$ preserving each $I_{\alpha}$ with its orientation. If $I_{\alpha}=X_{\gamma}$ for some $\gamma \in \Gamma$, then we set

$$
h_{\lambda}=\phi_{\gamma} \circ \sigma_{\gamma}^{-1}: X_{\gamma} \rightarrow X_{\gamma},
$$

otherwise put $h_{\lambda}$ to be the identity map $\mathrm{id}_{I_{\alpha}}$.

Then $h_{\lambda}$ satisfies assumptions of Theorem 2.3 and therefore extends to a foliated homeomorphism $h_{\lambda}: S_{\lambda} \rightarrow S_{\lambda}$. Hence we get a homeomorphism $h: Z_{0} \rightarrow Z_{0}$ defined by $\left.h\right|_{S_{\lambda}}=h_{\lambda}$.

Then one easily checks that the map $q^{\prime}=q \circ h: Z_{0} \rightarrow Z$ is a striped atlas for $Z$. Moreover, $q^{\prime}$ glues the same strips along the same boundary intervals and in the same directions as $q$, but its gluing maps

$$
\phi_{\gamma}^{\prime}=\left.\left(\left.q^{\prime}\right|_{X_{\gamma}}\right)^{-1} \circ q^{\prime}\right|_{Y_{\gamma}}: Y_{\gamma} \rightarrow X_{\gamma}
$$

differs from the ones of $q$. It follows from the following commutative diagram:

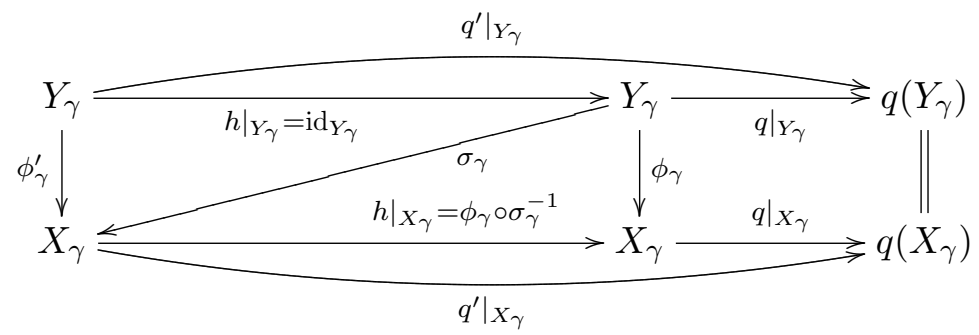

that $\phi_{\gamma}^{\prime}=\sigma_{\gamma}$ is affine. Hence $q^{\prime}$ is an affine striped atlas for $Z$.

\section{Graph of a StRIPED atlas}

Let $q: \underset{\lambda \in \Lambda}{\sqcup} S_{\lambda} \rightarrow Z$ be a striped atlas on $Z$. We will now associate to $q$ a certain graph $G$ which encodes a "combinatorial" information about gluing strips via $q$. It was firstly considered in [12] for a special class of "rooted tree like" striped surfaces. That graph may have multiple edges and loops and also half-open edges.

(1) The vertices of $G$ are strips of $\underset{\lambda \in \Lambda}{\sqcup} S_{\lambda}$. 
(2) It will be convenient to call each boundary interval $X$ of some strip $S_{\lambda}$ a half-edge incident to the vertex $S_{\lambda}$. The set of all half edges of $\partial_{ \pm} S_{\lambda}$ will be denoted by $d_{ \pm}\left(S_{\lambda}\right)$. We also put $d\left(S_{\lambda}\right)=d_{-}\left(S_{\lambda}\right) \cup d_{+}\left(S_{\lambda}\right)$.

(3) The edges of $G$ are of the following two types.

(a) If two strips $S_{1}$ and $S_{2}$ are glued along their boundary intervals $X_{\gamma}$ and $Y_{\gamma}$, then we assume that the vertices $S_{1}$ and $S_{2}$ of $G$ are connected by an edge $e_{\gamma}$. Thus formally, an edge $e_{\gamma}$ is an unordered pair of half edges $\left(X_{\gamma}, Y_{\gamma}\right)$ and will be called a closed edge of $G$.

(b) If $X$ is a boundary interval of some strip $S_{\lambda}$ which is not glued to any other interval, so it represents a boundary interval of $Z$, then we assume that $X$ is a half-open edge with one vertex $S_{\lambda}$.

We also add to $G$ the information about directions of gluing boundary intervals, and the disposition of boundary intervals along each strip.

For a homeomorphism $f:(a, b) \rightarrow(c, d)$ define a number or $(f)=+1$ if $f$ preserves orientation and or $(f)=-1$ otherwise. It is evident, that if $g:(c, d) \rightarrow(e, f)$ is another homeomorphism, then or $(g \circ f)=\operatorname{or}(g) \cdot \operatorname{or}(f)$.

(4) To each closed edge $\left(X_{\gamma}, Y_{\gamma}\right)$ corresponding to the gluing of boundary components $\phi_{\gamma}: Y_{\gamma} \rightarrow X_{\gamma}$ we associate the number $\sigma\left(X_{\gamma}, Y_{\gamma}\right):=$ $\operatorname{or}\left(\phi_{\gamma}\right)$ and call it the orientation of gluing.

(5) Recall that for each strip $S_{\lambda}$ the set of its boundary intervals is at most countable partially ordered set being a disjoint union of two linearly ordered subsets corresponding to $\partial_{-} S_{\lambda}$ and $\partial_{+} S_{\lambda}$ respectively. Therefore we have a linear order on each of the sets $d_{-}\left(S_{\lambda}\right)$ and $d_{+}\left(S_{\lambda}\right)$ of all half-edges incident to the vertex $S_{\lambda}$ of $G$.

Thus, "very formally", a graph of a striped atlas is the following object

$$
G=(\Lambda, H, \xi, \sigma)
$$

where

- $\Lambda$ is a set, called the set of vertices of $G$.

- $H=\bigsqcup_{\lambda \in \Lambda}\left(d_{-1}(\lambda) \sqcup d_{+1}(\lambda)\right)$ is a family of mutually disjoint at most countable linearly ordered sets, $d_{-1}(\lambda)$ and $d_{+1}(\lambda)$, called half-edges incident to $\lambda$. We also denote $d(\lambda)=d_{-}(\lambda) \sqcup d_{+}(\lambda)$.

- $\xi: H \rightarrow H$ is an involution, i.e. a bijection such that $\xi^{2}=\mathrm{id}_{H}$. In this case if $X \neq \xi(X)$ for some $X \in H$, then the unordered pair $\{X, \xi(X)\}$ is called a closed edge of $G$. Otherwise $X$ is fixed point of $\xi$ and is called a half-open edge of $G$.

- $\sigma: E \rightarrow\{ \pm 1\}$ is a map from the set

$$
E=\{\{X, \xi(X)\} \mid X \in H, X \neq \xi(X)\}
$$

of all closed edges of $G$ to $\{ \pm 1\}$, called orientation of gluing. 
Equivalently, $\sigma$ can be regarded as a map $\sigma: H \backslash \operatorname{Fix}(\xi) \rightarrow\{ \pm 1\}$ such that $\sigma \circ \xi=\sigma$.

Definition 6.1. Let $G=(\Lambda, H, \xi, \sigma)$ and $G^{\prime}=\left(\Lambda^{\prime}, H^{\prime}, \xi^{\prime}, \sigma^{\prime}\right)$ be graphs of striped atlases of some striped surfaces. Then by an isomorphism of these graphs we will mean four maps

$$
\nu: \Lambda \rightarrow \Lambda^{\prime}, \quad \varepsilon: H \rightarrow H^{\prime}, \quad l, \tau: \Lambda \rightarrow\{ \pm 1\},
$$

having the following properties.

(a) $\nu$ and $\varepsilon$ are bijections satisfying the identity

$$
\varepsilon\left(d_{s}(\lambda)\right)=d_{\tau(\lambda) \cdot s}^{\prime}(\nu(\lambda))
$$

for all $\lambda \in \Lambda$ and $s \in\{ \pm 1\}$, where $d_{ \pm 1}^{\prime}\left(\lambda^{\prime}\right) \subset H^{\prime}$ is the set of half edges of $G^{\prime}$ incident to $\lambda^{\prime} \in \Lambda$. Moreover, both bijections

$$
\begin{aligned}
& \left.\varepsilon\right|_{d_{-1}(\lambda)}: d_{-1}(\lambda) \rightarrow d_{-\tau(\lambda)}^{\prime}(\nu(\lambda)), \\
& \left.\varepsilon\right|_{d_{+1}(\lambda)}: d_{+1}(\lambda) \rightarrow d_{\tau(\lambda)}^{\prime}(\nu(\lambda)),
\end{aligned}
$$

are increasing for $l(\lambda)=+1$ and decreasing for $l(\lambda)=-1$.

(b) $\xi^{\prime} \circ \varepsilon=\varepsilon \circ \xi$, in particular, $\varepsilon$ induces a bijection between closed edges of $G$ and $G^{\prime}$.

(c) Let $\{X, Y\}$ be a closed edge of $G$ with $X \in d(\lambda)$ and $Y=\xi(X) \in d(\mu)$ for some $\lambda, \mu \in \Lambda$. Then

$$
l(\lambda) \cdot \sigma(X, Y)=\sigma^{\prime}(\varepsilon(X), \varepsilon(Y)) \cdot l(\mu) .
$$

Notice that the set $\operatorname{Aut}(G)$ of all automorphisms of a graph $G$ is a group with respect to the following multiplication: if

$$
a^{\prime}=\left(\nu^{\prime}, \varepsilon^{\prime}, l^{\prime}, \tau^{\prime}\right), a=(\nu, \varepsilon, l, \tau) \in \operatorname{Aut}(G),
$$

then their product $a^{\prime \prime}=a^{\prime} a=\left(\nu^{\prime \prime}, \varepsilon^{\prime \prime}, l^{\prime \prime}, \tau^{\prime \prime}\right)$ is defined as follows:

$$
\begin{aligned}
\nu^{\prime \prime} & =\nu^{\prime} \circ \nu, & \varepsilon^{\prime \prime} & =\varepsilon^{\prime} \circ \varepsilon, \\
l^{\prime \prime}(\lambda) & =l^{\prime}(\nu(\lambda)) \cdot l(\lambda), & \tau^{\prime \prime}(\lambda) & =\tau^{\prime}(\nu(\lambda)) \cdot \tau(\lambda),
\end{aligned}
$$

for all $\lambda \in \Lambda$.

Let $1: \Lambda \rightarrow\{ \pm 1\}$ be the constant function taking value +1 . Then $\left(\operatorname{id}_{\Lambda}, \operatorname{id}_{H}, \mathbf{1}, \mathbf{1}\right)$ is the unit of $\operatorname{Aut}(G)$ and $(\nu, \varepsilon, l, \tau)^{-1}=\left(\nu^{-1}, \varepsilon^{-1}, l, \tau\right)$.

For a set $X$ denote by $\Sigma(X)$ the group of all bijections of $X$, that is the permutation group on $X$. For a group $A$ let also $A^{X}$ be the group of all maps $X \rightarrow A$ with respect to the point-wise multiplication. Then the group $\Sigma(X)$ naturally acts from the right on $A^{X}$ by the rule: the result of the action of a bijection $\nu: X \rightarrow X$ from $\Sigma(X)$ on a map $a: X \rightarrow A$ belonging to $A^{X}$ is the composition map

$$
a \circ \nu: X \stackrel{\nu}{\longrightarrow} X \stackrel{a}{\longrightarrow} A \text {. }
$$


The corresponding semidirect product $A^{X} \rtimes \Sigma(X)$ is called the wreath product of $\Sigma(X)$ and $A$ over $X$ and denoted by $A 2_{X} \Sigma(X)$. Thus, by definition, $A \imath_{X} \Sigma(X)$ is a direct product of sets $A^{X} \times \Sigma(X)$ with respect to the following multiplication:

$$
\left(a^{\prime}, \nu^{\prime}\right)(a, \nu)=\left(\left(a^{\prime} \circ \nu\right) \cdot a, \nu^{\prime} \circ \nu\right),
$$

where $\cdot$ denotes multiplication in $A^{X}$. Notice that there is a natural surjective homomorphism $\eta: A l_{X} \Sigma(X) \rightarrow \Sigma(X), \eta(a, \nu)=\nu$, whose kernel is $A^{X} \times \mathrm{id}_{X}$. Moreover, we also have an inclusion $1 \times \Sigma(X) \subset A$ ? $_{X} \Sigma(X)$, where $1: X \rightarrow A$ is the constant map into the unit of $A$. In other words the following short exact sequence

$$
1 \rightarrow A^{X} \rightarrow A 2_{X} \Sigma(X) \stackrel{\eta}{\longrightarrow} \Sigma(X) \rightarrow 1
$$

admits a section $s: \Sigma(X) \rightarrow A \imath_{X} \Sigma(X), s(\nu)=(\mathbf{1}, \nu)$, i.e. a homomorphism such that $\eta \circ s=\mathrm{id}(\Sigma(X))$.

Rewriting (6.2) and (6.3) in the form:

$$
\left(\nu^{\prime}, \varepsilon^{\prime}, l^{\prime}, \tau^{\prime}\right)(\nu, \varepsilon, l, \tau)=\left(\nu^{\prime} \circ \nu, \varepsilon^{\prime} \circ \varepsilon,\left(l^{\prime} \circ \nu\right) \cdot l,\left(\tau^{\prime} \circ \nu\right) \cdot \tau\right)
$$

we see that $\operatorname{Aut}(G)$ is a subgroup of

$$
\left(\{ \pm 1\}^{2} \imath_{\Lambda} \Sigma(\Lambda)\right) \times \Sigma(H) .
$$

Theorem 6.2. Each equivalence of striped atlases induces an isomorphism between their graphs. Conversely, each isomorphism between their graphs is induced by some striped atlases equivalence.

Before proving Theorem 6.2 let us first consider several illustrating examples. To preserve the formalism we need to talk about maps from empty set. As usual, we identify a map $f: A \rightarrow B$ between sets with its graph $\{(a, f(a)) \mid a \in A\} \subset A \times B$. Therefore a map $\varnothing \rightarrow B$ from empty set is an empty subset of the empty set $\varnothing \times B$.

Example 6.3. Let $S=\mathbb{R} \times(-1,1)$ and $q=\operatorname{id}_{S}: S \rightarrow S$ be a striped atlas consisting of one strip, see Figure 6.1(a). Then $\Lambda=\{*\}$ consists of a unique point, $H=\varnothing$, and so $\xi: H \rightarrow H$ and $\sigma: H \backslash \operatorname{Fix}(\xi) \rightarrow\{ \pm 1\}$ are maps of empty set.

Let $(\nu, \varepsilon, l, \tau) \in \operatorname{Aut}(G)$. Then $\nu=\operatorname{id}_{\Lambda}$ and $\varepsilon=\operatorname{id}_{H}$ are uniquely determined, while $l, \tau:\{*\} \rightarrow\{ \pm 1\}$ can be arbitrary maps. It easily follows that $\operatorname{Aut}(G) \cong\{ \pm 1\} \times\{ \pm 1\}$.

Example 6.4. Let $S=\mathbb{R} \times(-1,1) \cup\{(-2,-1) \cup(1,2)\} \times\{1\}$ and again $q=\operatorname{id}_{S}: S \rightarrow S$ be a striped atlas consisting of one strip, see Figure 6.1(b). Then $\Lambda=\{*\}$ consists of a unique point, $H=\{a, b\}=d_{+1}(*)$, where $a=(-2,-1) \times\{1\}, b=(1,2) \times\{1\}$, and $a<b$ in the sense of the linear order 


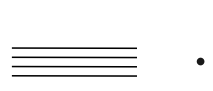

(a)

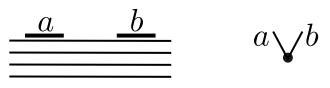

(b)

FIGURE 6.1. Striped atlases consisting of one strip and being the identity homeomorphisms

in $d_{+1}(*)$. As these intervals are not glued, we see that $\xi=\operatorname{id}_{H}: H \rightarrow H$ and so $\sigma: H \backslash \operatorname{Fix}(\xi) \rightarrow\{ \pm 1\}$ is a map from empty set.

Let $x=(\nu, \varepsilon, l, \tau) \in \operatorname{Aut}(G)$. Then $\nu=\mathrm{id}_{\Lambda}$. Moreover, as $H=d_{+1}(*)$, and so $d_{-1}(*)=\varnothing$, it follows that $\varepsilon\left(d_{+1}(*)\right)=d_{+1}(*)$, whence $\tau(*)=+1$.

If $\varepsilon(a)=a$, then $\varepsilon=\operatorname{id}_{H}$, whence $l(*)=+1$, and so $x$ is the unit of $\operatorname{Aut}(G)$. Suppose $\varepsilon(a)=b$, then $\varepsilon(b)=a$, so $\varepsilon$ is an order reversing bijection of $H=d_{+1}(*)$, whence $l(*)=-1$. Thus $\operatorname{Aut}(G)$ consists of two elements, i.e. $\operatorname{Aut}(G) \cong\{ \pm 1\}$.

Example 6.5. Let $S=\mathbb{R} \times[0,1], \phi: \mathbb{R} \times\{0\} \rightarrow \mathbb{R} \times\{+1\}$ be a homeomorphism given by $\phi(x, 0)=(x, 1)$, then the quotient $Z=S / \phi$ is an open cylinder $\mathbb{R} \times S^{1}$, and the quotient map $q: S \rightarrow Z$ is a striped atlas, see Figure 6.2(a).

In this case $\Lambda=\{*\}$ again consists of a unique point, $H=\{a, b\}$, where $a=\mathbb{R} \times\{0\}, b=\mathbb{R} \times\{1\}, \xi: H \rightarrow H$ is given $\xi(a)=b, \xi(b)=a$, and $\sigma: H \rightarrow\{ \pm 1\}$ is defined by $\sigma(a)=\sigma(b)=\operatorname{or}(\phi)=+1$.

Let $x=(\nu, \varepsilon, l, \tau) \in \operatorname{Aut}(G)$. Then $\nu=\mathrm{id}_{\Lambda}$. Moreover, since $G$ has a unique edge $\{a, b\}, \varepsilon$ preserves this edge, whence it follows from (6.1) that $l(a)=l(b)$.

Suppose $\varepsilon(a)=a$, then $\varepsilon=\operatorname{id}_{H}$, whence $\tau(*)=+1$. Otherwise, $\varepsilon(a)=b$, $\varepsilon(b)=a$, and $\tau(*)=-1$. Notice that in both of those cases, the common value $l(a)=l(b)$ can be taken arbitrary.

This implies that $\operatorname{Aut}(G) \cong\{ \pm 1\} \times\{ \pm 1\}$.

Example 6.6. Suppose as in the previous example $S=\mathbb{R} \times[0,1]$, but now $\phi: \mathbb{R} \times\{0\} \rightarrow \mathbb{R} \times\{+1\}$ is given by $\phi(x, 0)=(-x, 1)$, and so it reverses orientation. In this case the quotient $Z=S / \phi$ is an open Möbius band, see Figure 6.2(b). One easily check that $\operatorname{Aut}(G) \cong\{ \pm 1\} \times\{ \pm 1\}$ as well.

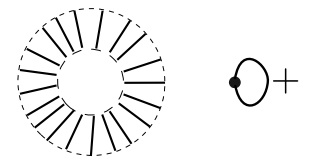

(a)

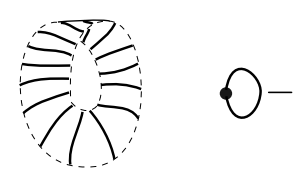

(b)

Figure 6.2. Foliated open cylinder and Möbius band 
Proof of Theorem 6.2. Let

$$
q: Z_{0}=\underset{\lambda \in \Lambda}{\sqcup} S_{\lambda} \rightarrow Z, \quad q^{\prime}: Z_{0}^{\prime}=\underset{\lambda^{\prime} \in \Lambda^{\prime}}{\sqcup} S_{\lambda^{\prime}}^{\prime} \rightarrow Z^{\prime}
$$

be striped atlases on surfaces $Z$ and $Z^{\prime}$ respectively, and $G=(\Lambda, H, \xi, \sigma)$ and $G^{\prime}=\left(\Lambda^{\prime}, H^{\prime}, \xi^{\prime}, \sigma^{\prime}\right)$ be the their graphs.

1) Suppose $(h, k)$ is a pair of homeomorphisms defining an equivalence of atlases, so we have a commutative diagram (5.2). Then $h$ induces a bijection between the connected components of $Z_{0}$ and $Z_{0}^{\prime}$ which yields a bijection $\nu: \Lambda \rightarrow \Lambda^{\prime}$ between the corresponding sets of indices (being in turn vertices of $G$ and $G^{\prime}$ ) such that $h\left(S_{\lambda}\right)=S_{\nu(\lambda)}^{\prime}$.

In particular, $h$ yields also a bijection between the boundary components of $Z_{0}$ and $Z_{0}^{\prime}$ being sets of half edges of $G$ and $G^{\prime}$. Thus we get a bijection $\varepsilon: H \rightarrow H^{\prime}$.

It remains to define the functions $l, \tau: \Lambda \rightarrow\{ \pm 1\}$. Take $\lambda \in \Lambda$ and consider the restriction $\left.h\right|_{S_{\lambda}}: S_{\lambda} \rightarrow S_{\nu(\lambda)}^{\prime}$. Assume that Int $S_{\lambda}=\mathbb{R} \times(a, b)$ and $\operatorname{Int} S_{\nu(\lambda)}=\mathbb{R} \times(c, d)$ for some $a<b, c<d \in \mathbb{R}$. Since $\left.h\right|_{S_{\lambda}}$ preserves leaves being horizontal lines, we have that

$$
\left.h\right|_{S_{\lambda}}(x, y)=(\alpha(x, y), \beta(y))
$$

where

- $\alpha: S_{\lambda} \rightarrow \mathbb{R}$ is a continuous function such that for each $y \in(a, b)$ the correspondence $x \mapsto \alpha(x, y)$ is a homeomorphism $\alpha_{y}: \mathbb{R} \rightarrow \mathbb{R}$;

- $\beta:(a, b) \rightarrow(c, d)$ is a homeomorphism.

Evidently, all homeomorphisms $\alpha_{y}$ are increasing or decreasing mutually for all $y \in(a, b)$, i.e. $\operatorname{or}\left(\alpha_{y}\right)$ does not depend on $y \in(a, b)$. Therefore we set

$$
l(\lambda)=\operatorname{or}\left(\alpha_{y}\right), \quad \tau(\lambda)=\operatorname{or}(\beta) .
$$

We claim that $(\nu, \varepsilon, l, \tau)$ is an isomorphism between graphs $G$ and $G^{\prime}$ in the sense of Definition 6.1.

Notice that the restriction $\left.h\right|_{\partial S_{\lambda}}: \partial S_{\lambda} \rightarrow \partial S_{\nu(\lambda)}^{\prime}$ is a monotone homeomorphism which easily implies conditions (a) and (b) of Definition 6.1. We leave the verification for the reader and will check condition (c) only.

Let $\{X, Y\}$ be a closed edge of $G$ with $X \in d(\lambda)$ and $Y=\xi(X) \in d(\mu)$ for some $\lambda, \mu \in \Lambda$, and $X^{\prime}=\varepsilon(X)$ and $Y^{\prime}=\varepsilon(Y)$. This means that $X \subset \partial S_{\lambda}$ and $Y \subset \partial S_{\mu}$ are boundary components with $q(X)=q(Y)$, $X^{\prime}=h(X) \subset \partial S_{\nu(\lambda)}^{\prime}$, and $Y^{\prime}=h(Y) \subset \partial S_{\nu(\mu)}^{\prime}$. Then we have the following 
commutative diagram:

$$
\begin{gathered}
Y \underset{\text { or }\left(\left.h\right|_{Y}\right)=l(\mu)}{\left.h\right|_{Y}} Y^{\prime} \\
\left.\phi \downarrow \operatorname{or}(\phi)=\sigma(X, Y) \quad \operatorname{or}\left(\phi^{\prime}\right)=\sigma^{\prime}\left(X^{\prime}, Y^{\prime}\right)\right\rfloor \phi^{\prime} \\
X \underset{\operatorname{or}\left(\left.h\right|_{X}\right)=l(\lambda)}{\left.h\right|_{X}} X^{\prime}
\end{gathered}
$$

where $\phi$ and $\phi^{\prime}$ are gluing homeomorphisms. Hence

$$
\begin{aligned}
l(\lambda) \cdot \sigma(X, Y) & =\operatorname{or}\left(\left.h\right|_{Y}\right) \cdot \operatorname{or}(\phi)=\operatorname{or}\left(\left.\phi \circ h\right|_{Y}\right)= \\
& =\operatorname{or}\left(\left.h\right|_{X} \circ \phi^{\prime}\right)=\operatorname{or}\left(\left.h\right|_{X}\right) \cdot \operatorname{or}\left(\phi^{\prime}\right)=l(\mu) \cdot \sigma^{\prime}\left(X^{\prime}, Y^{\prime}\right) .
\end{aligned}
$$

2) To prove the converse statement, notice that due to Theorem 5.8, one can assume in addition that both atlases $q$ and $q^{\prime}$ are affine.

Let $(\nu, \varepsilon, l, \tau)$ be an isomorphism between $G$ and $G^{\prime}$ in the sense of Definition 6.1.

Let $\lambda \in \Lambda$ and $\lambda^{\prime}=\nu(\lambda)$. We will now construct a homeomorphism $h_{\lambda}: S_{\lambda} \rightarrow S_{\lambda^{\prime}}^{\prime}$ in the following way.

(i) First suppose $\partial S_{\lambda}=\varnothing$, that is $d(\lambda)=\varnothing$. Since $\varepsilon$ bijectively maps $d(\lambda)$ onto $d^{\prime}\left(\lambda^{\prime}\right)$, it follows that $d^{\prime}\left(\lambda^{\prime}\right)=\varnothing$, and so $\partial S_{\nu(\lambda)}^{\prime}=\varnothing$ as well. Not loosing generality, one can assume that $S_{\lambda}=S_{\lambda^{\prime}}^{\prime}=\mathbb{R} \times(-1,1)$. Then we define $h_{\lambda}$ by the formula:

$$
h_{\lambda}(x, y)=(l(\lambda) x, \tau(\lambda) y) .
$$

(ii) Now assume that $\partial S_{\lambda} \neq \varnothing$. Let $X \in d(\lambda)$ be a half-edge in $G$ incident to the vertex $\lambda$, that is $X$ is a boundary component of $S_{\lambda}$. Then $\nu(X)$ is a boundary interval of $S_{\nu(\lambda)}^{\prime}$. Since we assumed that strips $S_{\lambda}$ and $S_{\nu(\lambda)}^{\prime}$ are model, the intervals $X$ and $\nu(X)$ are bounded. Define $h_{\lambda}$ on $X$ to be a unique affine homeomorphism $\psi_{X}: X \rightarrow \nu(X)$ with $\operatorname{or}\left(\psi_{X}\right)=l(\lambda)$.

The family of all $\left\{\psi_{X}\right\}_{X \in d(\lambda)}$ give a homeomorphism $h_{\lambda}: \partial S_{\lambda} \rightarrow \partial S_{\nu(\lambda)}^{\prime}$. Due to property (a) of Definition $6.1, h_{\lambda}$ is monotone, and therefore by Theorem $2.3 h$ extends to a foliated homeomorphism $h_{\lambda}: S_{\lambda} \rightarrow S_{\nu(\lambda)}^{\prime}$.

Thus we obtain a foliated homeomorphism $h: \underset{\lambda \in \Lambda}{\sqcup} S_{\lambda} \rightarrow \underset{\lambda^{\prime} \in \Lambda^{\prime}}{\sqcup} S_{\lambda^{\prime}}^{\prime}$ defined by $\left.h\right|_{S_{\lambda}}=h_{\lambda}$ for $\lambda \in \Lambda$.

We claim that $h$ induces a foliated homeomorphism $k: Z \rightarrow Z^{\prime}$ such that the pair $(h, k)$ is an equivalence of striped atlases $q$ and $q^{\prime}$.

Let $D \subset Z_{0}$, (resp. $D^{\prime} \subset Z_{0}^{\prime}$ ), be the set of boundary intervals on which $q$, (resp. $q^{\prime}$ ), is not injective. Then $h$ yields a homeomorphism of $Z_{0} \backslash D$ onto $Z_{0}^{\prime} \backslash D^{\prime}$, whence the restriction $k: q\left(Z_{0} \backslash D\right) \rightarrow q^{\prime}\left(Z_{0}^{\prime} \backslash D^{\prime}\right)$ must be given by $k=q^{\prime} \circ h \circ q^{-1}$. 
Therefore it remains to show that $h$ is "compatible" with $q$ and $q^{\prime}$ on $D$ and $D^{\prime}$ in the sense that for each pair of boundary intervals $X \subset \partial S_{\lambda}$ and $Y \subset \partial S_{\mu}$ with $q(X)=q(Y)$, we have that $q^{\prime}(h(X))=q^{\prime}(h(Y))$ and the following commutative diagram holds true:

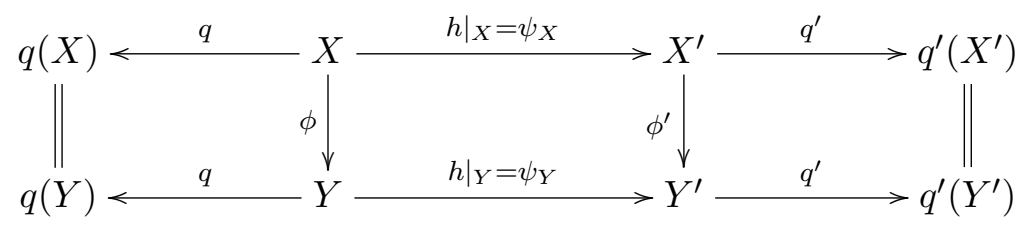

where $\phi$ and $\phi^{\prime}$ are gluing homeomorphisms. Then for each $z \in D$ we will set $k(q(z))=q^{\prime} \circ h(z)$.

In term of graphs we have that $\{X, Y\}$ is a closed edge of $G$ such that $X \in d(\lambda), Y=\xi(X) \in d(\mu), X^{\prime}=\varepsilon(X)$, and $Y^{\prime}=\varepsilon(Y)$. Then by (b)

$$
Y^{\prime}=\varepsilon(Y)=\varepsilon \circ \xi(X)=\xi^{\prime} \circ \varepsilon(X)=\xi^{\prime}(X)
$$

and so $\left\{X^{\prime}, Y^{\prime}\right\}$ is a closed edge of $G^{\prime}$, that is $q^{\prime}(h(X))=q^{\prime}(h(Y))$.

Then we have the diagram (6.4) but need to check commutativity of its central square consisting of affine homeomorphisms. It follows from (c) that $\operatorname{or}\left(\phi^{\prime} \circ \psi_{X}\right)=\operatorname{or}\left(\psi_{Y} \circ \phi\right)$. Since $\phi^{\prime} \circ \psi_{X}, \psi_{Y} \circ \phi: X \rightarrow Y^{\prime}$ are affine homeomorphisms, it follows that they coincide, and so diagram (6.4) is commutative.

Thus $(h, k)$ is an equivalence of striped atlases inducing given isomorphism $(\nu, \varepsilon, l, \tau)$ between $G$ and $G^{\prime}$.

\section{Characterization of a CERTAin Class of StRiped Surfaces}

Let $(Z, \Delta)$ be a foliated surface with countable base, $Z / \Delta$ the set of leaves of $\Delta$, and $p: Z \rightarrow Z / \Delta$ be the quotient map. We will endow $Z / \Delta$ with the quotient topology, so a subset $V \subset Z / \Delta$ is open if and only if $p^{-1}(V)$ is open in $Z$. Notice that a priori $Z / \Delta$ is not even a $T_{0}$-space.

For each leaf $\omega$ of $\Delta$ let $J_{\omega}=[0,1)$ if $\omega \subset \partial Z$ and $J_{\omega}=(-1,1)$ otherwise. Then a cross-section of $\Delta$ passing through $\omega$ is a continuous map $\gamma: J_{\omega} \rightarrow Z$ such that $\gamma(0) \in \omega$ and for distinct $s, t \in J_{\omega}$ their images $\gamma(s)$ and $\gamma(t)$ belong to distinct leaves of $\Delta$.

A subset $U \subset Z$ is called saturated if it is a union of leaves. For each leaf $\omega \in \Delta$ denote by $\mathrm{c}(\omega)$ the intersection of closures of all saturated neighbourhoods of $\omega$. Evidently $\omega \subset \mathrm{c}(\omega)$.

Definition 7.1. [8] A leaf $\omega$ will be called special whenever $\omega \neq \mathrm{c}(\omega)$, see Figure 7.1. We will denote by $\Sigma$ the family of all special leaves of $\Delta$.

In [4] and [3] special leaves were called branch points of $Z / \Delta$. 


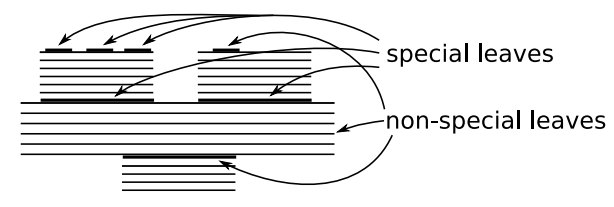

Figure 7.1. Special and non-special leaves

Lemma 7.2. Suppose there exist a striped atlas $q: \underset{\lambda \in \Lambda}{\sqcup} S_{\lambda} \rightarrow Z$ such that $\Delta$ is its canonical foliation. Let also $D=q\left(\underset{\lambda \in \Lambda}{\sqcup} \partial S_{\lambda}\right)$ be the union of images of boundary components of strips. Then

(i) $\Sigma \cup \partial Z \subset D$;

(ii) $\Sigma, \partial Z$, and $D$ are a locally finite families of leaves;

(iii) $\Sigma, \partial Z$, and $D$ are closed subsets of $Z$.

Proof. (i) By definition $\partial Z \subset D$. Moreover, one easily check that a leaf $\omega \in \Delta$ is special, i.e. $\omega \subset \Sigma$, if and only if there exists a boundary interval $X \subset \partial_{\epsilon} S_{\lambda}$ for some $\lambda \in \Lambda$ and $\epsilon \in\{ \pm\}$ such that $q(X)=\omega$ and $X \neq \partial_{\epsilon} S_{\lambda}$. Hence $\Sigma \subset D$ as well.

(ii). Evidently, each leaf $\omega$ in $D$ has an open neighbourhood containing no other leaves from $D$. This implies that $D$ is a locally finite family of closed subsets of $Z$, whence so any subfamily of $D$. In particular, this holds for $\Sigma$ and $\partial Z$.

(iii) follows from (ii), since each leaf of $\Delta$ is a closed subset of $Z$.

A striped atlas on $Z$ will be called reduced whenever $D=\Sigma \cup \partial Z$.

Theorem 7.3. [8, Theorem 3.7]. Let $Z$ be a striped surface with countable base. Then one of the following statements holds true: either

(1) $Z$ is foliated homeomorphic to the open cylinder or Möbius band from Examples 6.5 and 6.6, or

(2) $Z$ admits a reduced atlas.

Idea of proof. We briefly discuss the proof in terms of the graph $G$ of the striped atlas $q$. It will be convenient to say that an edge $\{X, Y\}$ of $G$ is unessential whenever $X=\partial_{\epsilon} S$ and $Y=\partial_{\epsilon^{\prime}} S^{\prime}$ for some distinct strips $S, S^{\prime}$ of the atlas and some $\epsilon, \epsilon^{\prime} \in\{ \pm\}$, see Figure 7.2.

In particular, each unessential edge corresponds to a non-special leaf $\omega \subset D \backslash(\Sigma \cup \partial Z)$. The principal observation of Theorem 7.3 is that gluing $S$ and $S^{\prime}$ along $X$ and $Y$ gives again a strip $S_{1}$, see [8, Lemma 3.2]. Therefore one can replace $S$ and $S^{\prime}$ in the atlas $q$ with $S_{1}$. 


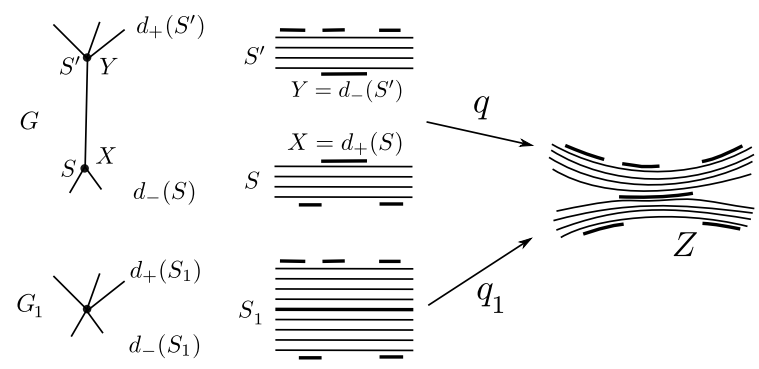

FigURE 7.2. Reduction of non-special leaves from $D$

On the graph this means that we replace a closed edge between $S$ and $S^{\prime}$ with one vertex. That techniques also allows to eliminate even countable paths of such edges. Hence if $G$ does not contain finite cycles of unessential edges, then, using the assumption that $Z$ has a countable base, one can remove all unessential edges and obtain a reduced atlas.

However, if there is a finite cycle of unessential edges, then one can remove all of them but one. This gives two special surfaces: open cylinder and Möbius band from Examples 6.5 and 6.6 in which we glue $X=\partial_{-} S$ with $Y=\partial_{+} S$. But the corresponding closed edge $\{X, Y\}$ is not unessential, since now $X$ and $Y$ belong to the same strip.

Consider the following five conditions on $(Z, \Delta)$.

(StrAtlas): $Z$ admits a striped atlas whose canonical foliation is $\Delta$.

( $\Sigma$ LocFin): The family $\Sigma$ of all special leaves of $\Delta$ is locally finite.

(PrjLocTriv): The quotient map $p: Z \rightarrow Z / \Delta$ is a locally trivial fibration and the space of leaves $Z / \Delta$ is locally homeomorphic with $[0,1)$ (though it is not in general a Hausdorff space).

(SatNbh): For each leaf $\omega \in \Delta$ there exist an open $\Delta$-saturated neighbourhood $U$ of $\omega$ and a homeomorphism $\eta: \mathbb{R} \times J_{\omega} \rightarrow U$ such that $\eta(\mathbb{R} \times t)$ is a leaf of $\Delta$ and $\eta(\mathbb{R} \times 0)=\omega$.

(CrossSect): Each leaf $\omega \in \Delta$ has a cross-section passing through $\omega$.

The following statement summarizes relations between the above properties obtained in [8], [9], [10], and in the present paper.

Theorem 7.4. [9], [10]. Let $(Z, \Delta)$ be a foliated surface satisfying the following two conditions:

(i) each leaf of $\Delta$ is a non-compact closed subset of $Z$;

(ii) each boundary component of $Z$ is a leaf of $\Delta$.

Then we have the following implications: 
- $($ PrjLocTriv $) \Rightarrow($ SatNbh $)+($ CrossSect $) ;$

- $($ StrAtlas $) \Rightarrow($ LLocFin $) \Rightarrow[($ PrjLocTriv $) \Leftrightarrow($ SatNbh $) \Leftrightarrow($ CrossSect $)]$;

- $(\Sigma$ LocFin $)+($ SatNbh $) \Rightarrow($ StrAtlas $)$.

In particular, if either (SatNbh) or (CrossSect) hold, then the conditions

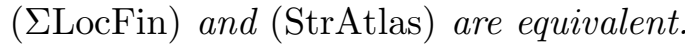

Proof. The implication (PrjLocTriv) $\Rightarrow$ (SatNbh) + (CrossSect) and the equivalence of conditions (SatNbh), (PrjLocTriv), and (CrossSect) under assumption ( $\Sigma$ LocFin) is proved in [9, Theorem 2.8].

The implication (StrAtlas) $\Rightarrow(\Sigma$ LocFin) is contained in statement (ii) of Lemma 7.2.

Finally, the implication $(\Sigma$ LocFin $)+($ SatNbh $) \Rightarrow$ (StrAtlas) is established in [10, Theorem 1.8].

Remark 7.5. For a striped surface $(Z, \Delta)$ with a striped atlas $q$ condition (SatNbh) is equivalent to the requirement that $q$ does not glue together boundary intervals belonging to the same side of the same strip. More precisely, if $q(X)=q(Y)$ for some distinct boundary intervals $X \subset \partial_{\epsilon} S$ and $Y \subset \partial_{\epsilon^{\prime}} S^{\prime}$, then either $S \neq S^{\prime}$ or $\epsilon \neq \epsilon^{\prime}$.

7.6. Foliated surface that does not admit a striped atlas. Consider the sequence $z_{n}=\left(0, \frac{1}{n}\right), n \in \mathbb{N}$, of points of $y$-axis on the plane converging to the origin $O$ and put $K=\left\{z_{n}\right\}_{n \in \mathbb{N}} \cup O$. Let also $Z=\mathbb{R}^{2} \backslash K$. Then $Z$ admits a foliation $\Delta$ into non-compact leaves being connected components of the intersection of $Z$ with horizontal lines.

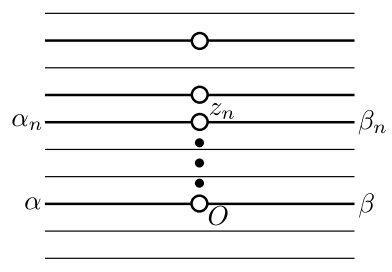

FiguRE 7.3.

Lemma 7.6.1. The pair $(Z, \Delta)$ satisfies condition (CrossSect) and violates ( $\Sigma$ LocFin). Hence it also violates (StrAtlas), that is $Z$ does not admit a striped atlas for which $\Delta$ is a canonical foliation.

Proof. (CrossSect). For each leaf $\omega \in \Delta$ there exists a cross-section being just an one vertical interval in $Z$ transversal to $\omega$.

To show that ( $\Sigma$ LocFin) fails, denote

$$
\alpha_{n}=(-\infty, 0) \times z_{n}, \quad \beta_{n}=(0,+\infty) \times z_{n},
$$




$$
\alpha=(-\infty, 0) \times O, \quad \beta=(0,+\infty) \times O .
$$

Then $\Sigma=\left\{\alpha_{n}, \beta_{n}\right\}_{n \in \mathbb{N}} \cup\{\alpha, \beta\}$ is the family of all special leaves of $\Delta$. Evidently,

$$
\mathrm{c}\left(\alpha_{n}\right)=\mathrm{c}\left(\beta_{n}\right)=\left\{\alpha_{n}, \beta_{n}\right\}, n \in \mathbb{N}, \quad \mathrm{c}(\alpha)=\mathrm{c}(\beta)=\{\alpha, \beta\},
$$

whence $\Sigma$ is not locally finite, since $\alpha_{n}$ converges to $\alpha$ and $\beta_{n}$ converges to $\beta$. Therefore by Theorem $7.4 Z$ does not admit a striped atlas with a canonical foliation $\Delta$.

Notice also that $Z^{\prime}=Z \backslash\{\alpha, \beta\}$ is disconnected and each of its connected components admits a striped atlas.

7.7. Foliation on the plane that does not admit a striped atlas. We will construct a more complicated example on the plane $\mathbb{R}^{2}$. Consider the foliation $\Delta_{0}$ on the strip $S=\mathbb{R} \times[0,1]$ shown in Figure 7.4(a). As indicated in Figure 7.4(b) it is glued from four strips. For each $k \in \mathbb{N}$

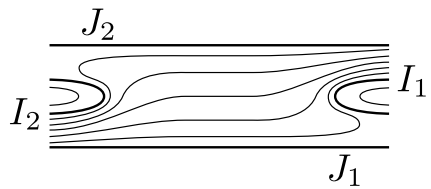

(a)

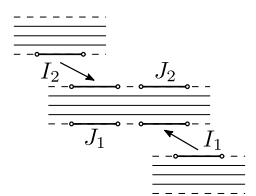

(b)

FIGURE 7.4 .

define the following strip $S_{k}=\mathbb{R} \times\left[\frac{1}{2^{k}}, \frac{1}{2^{k-1}}\right]$ and a homeomorphism

$$
\phi_{n}: S \rightarrow S_{k}, \quad \phi_{k}(x, y)=\left(k x,(y+1) / 2^{k}\right),
$$

so it expands strip along $x$-axis and shrinks it along $y$-axis.

Denote by $\Delta_{k}$ foliation on $S_{k}$ being the image of $\Delta_{0}$ under $\phi_{k}$. Then the union of all $\Delta_{k}$ gives a foliation on $\mathbb{R} \times[0,1]$ which extends to the foliation on all of $\mathbb{R}^{2}$ by parallel lines $\mathbb{R} \times y$ for $y \in(-\infty, 0] \cup(1,+\infty)$. We will denote that foliation on $\mathbb{R}^{2}$ by $\Delta$.

Then $\Sigma=\left\{\phi_{k}\left(I_{1}\right), \phi_{k}\left(I_{2}\right), \phi_{k}\left(J_{1}\right)=\phi_{k+1}\left(J_{2}\right)\right\}_{k \in \mathbb{N}}$ is the family of all special leaves of $\Delta$. This set is not locally finite since the leaves $\phi_{k}\left(J_{1}\right)$ converge to the leaf $\mathbb{R} \times 0$. One easily check that $\Delta$ satisfies (CrossSect), whence by Theorem $7.4\left(\mathbb{R}^{2}, \Delta\right)$ does not admit a striped atlas.

\section{HOMEOTOPY GROUP OF A CANONICAL FOLIATION}

Let $Z$ be a connected striped surface with a canonical foliation $\Delta$. Denote by $\mathcal{H}(\Delta)$ the group of all foliated homeomorphisms of $(Z, \Delta)$. Thus, by definition, $\mathcal{H}(\Delta)$ consists of all homeomorphism $h: Z \rightarrow Z$ such that for each leaf $\omega \in \Delta$ its image $h(\omega)$ is a leaf of $\Delta$ as well. Endow $\mathcal{H}(\Delta)$ with the 
compact open topology and let $\mathcal{H}_{0}(\Delta)$ be the identity path component of $\mathcal{H}(\Delta)$. It consists of all homeomorphisms $h \in \mathcal{H}(\Delta)$ isotopic to $\operatorname{id}_{Z}$ in $\mathcal{H}(\Delta)$. Then $\mathcal{H}_{0}(\Delta)$ is a normal subgroup of $\mathcal{H}(\Delta)$ and the quotient $\mathcal{H}(\Delta) / \mathcal{H}_{0}(\Delta)$ can be identified with the set $\pi_{0} \mathcal{H}(\Delta)$ of all path components of $\mathcal{H}(\Delta)$, that is $\pi_{0} \mathcal{H}(\Delta)=\mathcal{H}(\Delta) / \mathcal{H}_{0}(\Delta)$. This group will be called the homeotopy group of the foliation $\Delta$.

Theorem 8.1. c.f. [8, Theorem 4.4]. Let $q: \underset{\lambda \in \Lambda}{\sqcup} S_{\lambda} \rightarrow Z$ be a reduced affine striped atlas on a connected surface $Z, G$ be its graph, and $\Delta$ be the corresponding canonical foliation.

(i) If $Z$ is foliated homeomorphic either to an open cylinder from Example 6.5 or a Möbius band from Example 6.6 then $\mathcal{H}_{\mathrm{id}}(\Delta)$ is homotopy equivalent to the circle $S^{1}$.

(ii) Otherwise, $\mathcal{H}_{\mathrm{id}}(\Delta)$ is contractible.

In all the cases we have an isomorphism $\rho: \pi_{0} \mathcal{H}(\Delta) \cong \operatorname{Aut}(G)$.

Proof. (i) Suppose $Z$ is either an open cylinder or a Möbius band. Since by Examples 6.5 and $6.6 \operatorname{Aut}(G) \cong\{ \pm 1\} \times\{ \pm 1\}$ we need only to show that $\mathcal{H}_{\text {id }}(\Delta)$ is homotopy equivalent to the circle and $\pi_{0} \mathcal{H}(\Delta) \cong\{ \pm 1\} \times\{ \pm 1\}$ as well. We leave this statement as an exercise for the reader.

(ii). Now let $Z$ be neither an open foliated cylinder nor a foliated Möbius band. Then the following statement is a reformulation of [8, Theorem 4.4] in terms of striped atlases and their graphs. In particular, it contains (ii).

Lemma 8.1.1. c.f. [8, Theorem 4.4]. For each $k \in \mathcal{H}(\Delta)$ there exists a unique homeomorphism $h: Z_{0} \rightarrow Z_{0}$ such that $q \circ h=k \circ q$, i.e. $(h, k)$ is a self-equivalence of the atlas $q$. Moreover, $k \in \mathcal{H}_{\mathrm{id}}(\Delta)$ if and only if $(h, k)$ induces the identity automorphism of $G$.

Also the group $\mathcal{H}_{\mathrm{id}}(\Delta)$ is contractible.

It remains to construct an isomorphism $\rho: \pi_{0} \mathcal{H}(\Delta) \cong \operatorname{Aut}(G)$. Let $k \in \mathcal{H}(\Delta)$ and $(h, k)$ be the self-equivalence of the atlas $q$. Denote by $\rho(k)$ the automorphism of $G$ induced by $(h, k)$, see Theorem 6.2. Then one easily check that the correspondence $k \mapsto \rho(k)$ is a homomorphism $\rho: \pi_{0} \mathcal{H}(\Delta) \cong \operatorname{Aut}(G)$.

Moreover, by Theorem $6.2 \rho$ is surjective, and by Lemma 8.1.1 its kernel is $\mathcal{H}_{\text {id }}(\Delta)$. This gives the required isomorphism

$$
\pi_{0} \mathcal{H}(\Delta)=\mathcal{H}(\Delta) / \mathcal{H}_{\mathrm{id}}(\Delta)=\mathcal{H}(\Delta) / \operatorname{ker}(\rho) \cong \operatorname{Aut}(G)
$$

Theorem 8.1 is completed. 


\section{REREFENCES}

[1] William M. Boothby. The topology of regular curve families with multiple saddle points. Amer. J. Math., 73:405-438, 1951.

[2] William M. Boothby. The topology of the level curves of harmonic functions with critical points. Amer. J. Math., 73:512-538, 1951.

[3] C. Godbillon, G. Reeb. Fibrés sur le branchement simple. Enseignement Math. (2), 12:277-287, 1966.

[4] André Haefliger, Georges Reeb. Variétés (non séparées) à une dimension et structures feuilletées du plan. Enseignement Math. (2), 3:107-125, 1957.

[5] James Jenkins, Marston Morse. Contour equivalent pseudoharmonic functions and pseudoconjugates. Amer. J. Math., 74:23-51, 1952.

[6] Wilfred Kaplan. Regular curve-families filling the plane, I. Duke Math. J., 7:154-185, 1940.

[7] Wilfred Kaplan. Regular curve-families filling the plane, II. Duke Math J., 8:11-46, 1941.

[8] Sergiy Maksymenko, Eugene Polulyakh. Foliations with non-compact leaves on surfaces. Proceedings of Geometric Center, 8(3-4):17-30, 2015.

[9] Sergiy Maksymenko, Eugene Polulyakh. Foliations with all non-closed leaves on noncompact surfaces. Methods Funct. Anal. Topology, 22(3):266-282, 2016.

[10] Sergiy Maksymenko, Eugene Polulyakh. One-dimensional foliations on topological manifolds. Proceedings of Geometric Center, 9(2):1-23, 2016.

[11] Marston Morse. The existence of pseudoconjugates on Riemann surfaces. Fund. Math., 39:269-287 (1953), 1952.

[12] Yuliya Soroka. Homeotopy groups of rooted tree like non-singular foliations on the plane. Methods Funct. Anal. Topology, 22(3):283-294, 2016.

[13] Yuliya Soroka. Homeotopy groups of nonsingular foliations of a plane. Ukrainian Mathematical Journal, 2017, to appear.

Received: January 23, 2017, accepted: Febuary 20, $201 \%$.

Sergiy Maksymenko

Institute of Mathematics of NAS of Ukraine, Tereshchenkivska str. 3, Kyiv, 01004, UKRAINE

Email: maks@imath.kiev.ua

ORCID: orcid.org/0000-0002-0062-5188

Eugene Polulyakh

Institute of Mathematics of NAS of Ukraine, Tereshchenkivska Str. 3, Kyiv, 01004, UKRAINE

Email: polulyah@imath.kiev.ua

Yuliya Soroka

TARAs Shevchenko National University of Kyiv

Email: ladyice09@gmail.com 